\title{
Experimental investigation of the effect of classical noise on quantum non-Markovian dynamics
}

\author{
Simone Cialdi, ${ }^{*}$ Claudia Benedetti $\odot$, Dario Tamascelli, Stefano Olivares, Matteo G. A. Paris, and Bassano Vacchini $\odot^{\dagger}$ \\ Quantum Technology Lab, Dipartimento di Fisica “Aldo Pontremoli," Università degli Studi di Milano, Via Celoria 16, I-20133 Milan, Italy \\ and INFN, Sezione di Milano, Via Celoria 16, I-20133 Milan, Italy
}

(Received 20 August 2019; published 6 November 2019)

\begin{abstract}
We provide an experimental study of the relationship between the action of different classical noises on the dephasing dynamics of a two-level system and the non-Markovianity of the quantum dynamics. The two-level system is encoded in the photonic polarization degrees of freedom and the action of the noise is obtained via a spatial light modulator, thus allowing for an easy engineering of different random environments. The quantum non-Markovianity of the dynamics driven by classical Markovian and non-Markovian noise, both Gaussian and non-Gaussian, is studied by means of the trace distance. Our study clearly shows the different nature of the notion of non-Markovian classical process and non-Markovian quantum dynamics.
\end{abstract}

DOI: 10.1103/PhysRevA.100.052104

\section{INTRODUCTION}

The characterization of quantum non-Markovian processes has recently attracted a lot of attention: besides its conceptual interest it might open the way to obtain improved performances in quantum thermodynamics [1], higher sensitivities in quantum metrology [2,3], and techniques for complex quantum systems probing [4]. A natural and intriguing question is the relationship between the proposed notions of quantum non-Markovian dynamics (see Refs. [5-7] for reviews) and the classical notion of memoryless or Markovian process. This parallel has been the object of different theoretical studies [8-13] and has indeed provided the motivation for one of the first approaches to the problem [14].

In this paper we address this question from a new viewpoint, relying on the experimental realization of quantum dynamics depending on a classical random process. In such a way we relate a classical input with a quantum output and investigate the features of the latter with respect to the former. To this aim we need to generate a wide variety of classical processes with known features. We perform this task by obtaining such stochastic processes as a solution of suitable stochastic differential equations, so that we can obtain both Gaussian and non-Gaussian, Markovian and non-Markovian classical processes.

The experimental implementation is based on a quantum optics setup which allows us to engineer in a controlled way a dephasing dynamics determined by a classical stochastic process, which affects the polarization degrees of freedom of photons. A suitable configuration allows us to address in parallel a high number of realizations of the process and average them automatically in the detection stage.

The paper is organized as follows: In Sec. II we introduce the model of the system and the environment used to relate a classical input with a quantum output. In Sec. III we discuss

\footnotetext{
*simone.cialdi@unimi.it

†bassano.vacchini@mi.infn.it
}

how to generate classical noise with known features. We introduce the experimental setup in Sec. IV and discuss the results obtained in Sec. V. We draw our conclusions and final remarks in Sec. VI.

\section{THE MODEL}

\section{A. Evolution map}

To investigate by means of experiment how the different hallmarks of classical noise, such as being Gaussian or Markovian, affect the features of quantum dynamics, especially in view of the property of non-Markovianity, we consider the following simple but versatile model: The Hamiltonian describing the time evolution of the system is given by

$$
H(t)=X(t) \hbar \omega_{0} \sigma_{z},
$$

where $X(t)$ denotes a classical stochastic process with independent increments, describing the effect of the environmental noise on the two-level system of interest, and $\omega_{0}$ denotes the natural energy splitting of the two-level system, fixing scale and dimensions. The time evolution operator determining the effect of each single realization of the noise reads

$$
U(t, 0)=e^{-i \omega_{0} \sigma_{z} \int_{0}^{t} d \tau X(\tau)},
$$

so that, upon averaging over the environmental influence, one obtains for the reduced system dynamics

$$
\rho_{S}(t)=\Lambda(t)\left[\rho_{S}(0)\right]=\mathbb{E}\left[U(t, 0) \rho_{S}(0) U(t, 0)^{\dagger}\right],
$$

where $\mathbb{E}[\cdot]$ denotes the expectation value over the sample space of the noise. We are interested in investigating the behavior of $\rho_{S}(t)$ in its dependence from the noise $X(t)$.

To this aim we denote by

$$
\mathcal{X}(t)=\int_{0}^{t} d \tau X(\tau)
$$

the integral over time of the stochastic process, leading to $U(t, 0)=\operatorname{diag}\left(e^{-i \omega_{0} \mathcal{X}(t)}, e^{+i \omega_{0} \mathcal{X}(t)}\right)$, so that the reduced system dynamics is fully captured by the transformation of the 
off-diagonal matrix element

$$
\rho_{S}^{10}(0) \stackrel{t}{\rightarrow} \rho_{S}^{10}(0) \mathbb{E}\left[\exp \left(-2 i \omega_{0} \mathcal{X}(t)\right)\right]
$$

This simple description allows for a high experimental freedom in the implementation of the environmental noise and a unique characterization of non-Markovianity of the ensuing quantum dynamics. Indeed the evolution corresponds to a pure dephasing dynamics, for which all definitions of quantum non-Markovianity coincide [5-7], so that a clear-cut signature of quantum memory effects can be provided. In such a way the considered model well serves the purpose of exploring the effects of different classical noises on a quantum dynamics.

\section{B. Features of quantum dynamics}

The signature of the quantum dynamics that we want to address, in its dependence on the noise describing the effect of the environment, is its non-Markovianity. In the model considered, once we fix the noise acting in the Hamiltonian, we obtain a quantum dynamics which, while being given by a unitary transformation for each fixed realization of the noise, provides a time-dependent collection of completely positive trace preserving maps upon averaging over the noise. This collection of maps describes a stochastic quantum dynamics, which would generally arise as a consequence of the interaction with a suitable quantum environment. Indeed, as has been recently considered, any classical average can be seen to arise from a microscopic description with ancillary quantum degrees of freedom initialized in a classical state [15]. In this respect the obtained dynamics provides a well-defined reduced quantum dynamics whose features can be studied in view of the relationship between the properties of the input noise and the features of the output maps. As a figure of merit we consider the non-Markovianity of the ensuing quantum dynamics as characterized by the behavior in time of the trace distance between two initially distinct system states. This viewpoint was first introduced in Refs. [16,17] and connected to a notion of information exchange between system and environment. In particular, it is important to stress that this physical intuition remains confirmed even if the map, as in this case, is obtained upon averaging with respect to a classical label. This is an important issue, which has been the object of different investigations [15,18,19]. For the present simple model, as already stressed, essentially all proposed definitions of non-Markovianity coincide [6] since the dynamics is captured by the transformation (3). The natural quantifier of non-Markovianity for this class of models is therefore the behavior of the quantity

$$
D[\{X(t)\}]=\left|\mathbb{E}\left[\exp \left(-2 i \omega_{0} \mathcal{X}(t)\right)\right]\right|
$$

which describes the dephasing effect of the environmental noise on the system dynamics. In the trace-distance formalism this estimator is obtained considering a pair of states which maximize the possible revivals of the considered quantity. A monotonic decrease in time of this quantity corresponds to a Markovian dynamics, while a non-Markovian dynamics is obtained if revivals in time appear.

\section{CLASSICAL NOISE GENERATION}

In considering classical stochastic processes, two classes stand out in their relevance for applications and theoretical treatments; namely, Gaussian and Markovian processes. In both cases a relatively simple description applies, at variance with the case of a generic process. As a matter of fact, while in the general case a description of the process calls for knowledge of all its correlation functions, fixing the probability for given outcomes of the random variable at given times, in the case of Gaussian and Markovian processes a drastic simplification applies. A Gaussian process is in fact fixed by first and second moments only, while a Markovian process is determined by initial probability distribution and transition probability [20]. It is therefore of interest to explore the effect of noise on a quantum dynamics classifying the classical noises with respect to these two distinctive features.

To the aim of generating in a simple way these different types of noise we consider as a starting point two Markovian processes whose realizations can be easily simulated. Our starting tools are therefore Wiener and random telegraph noise, both Markovian: Gaussian the former, non-Gaussian the latter. Stochastic processes $X(t)$ with different features to be used in the dynamics given by Eq. (1) will be obtained as solution of stochastic differential equations with different input noises.

As Markovian Gaussian process we consider an OrnsteinUhlenbeck process $X_{\mathrm{OU}}(t)$ with friction coefficient $\gamma$ and diffusion constant $\sigma$, which can be obtained as solution of a linear stochastic differential equation driven by Wiener noise. We will further denote as $X_{\mathrm{RTN}}(t)$ a random telegraph noise with switching rate $\gamma$ and step one, whose realizations therefore jump from plus to minus one according to a Poisson process with rate $\gamma$. Such a process is still Markovian, but its probability density is not in Gaussian form. Using the realization of these processes as input noise we can obtain noise with different features.

Let us first consider the equation

$$
d Y(t)=-\kappa Y(t) d t+d X_{\mathrm{OU}}(t)
$$

with $\kappa$ being a positive rate. The process $Y(t)$ is still Gaussian due to linearity of the equation but non-Markovian, since its determination requires the knowledge of $X_{\mathrm{OU}}(t)$ up to time $t$ $[21,22]$. Using the same strategy we can obtain the increments of a process which is neither Gaussian nor Markovian by considering the stochastic differential equation

$$
d Z(t)=-\mu Z(t) d t+d X_{\mathrm{RTN}}(t),
$$

with $\mu$ being a positive rate. Using as seeds Wiener and random telegraph noise we are thus able to generate via the stochastic differential equations given by Eqs. (5) and (6), increments of stochastic processes which share or lack the distinct features of Gaussianity and Markovianity according to the corresponding well-established classical definitions.

To estimate the effect of the different noises on the dynamics we further need to evaluate the expectation value of the integral over time of the considered noise, defined as in Eq. (3). The analytic evaluation of this quantity is only feasible in special cases. For the case of the Ornstein-Uhlenbeck 
process we define

$$
\mathcal{X}_{\mathrm{OU}}(t) \equiv \int_{0}^{t} d \tau X_{\mathrm{OU}}(\tau)
$$

and exploiting Gaussianity one obtains for the quantity determining the dephasing of the two-level system

$$
\begin{aligned}
D\left[\left\{X_{\mathrm{OU}}(t)\right\}\right] & \equiv\left|\mathbb{E}\left[\exp \left(-2 i \omega_{0} \mathcal{X}_{\mathrm{OU}}(t)\right)\right]\right| \\
& =\exp \left(-\frac{\omega_{0}^{2} \sigma^{2}}{\gamma^{3}}\left(2 \gamma t-3-e^{-2 \gamma t}+4 e^{-\gamma t}\right)\right),
\end{aligned}
$$

where $\gamma$ and $\sigma$ denote, respectively, the friction and the diffusion coefficient of the process.

For the case of random telegraph noise, defining on the same footing

$$
\mathcal{X}_{\mathrm{RTN}}(t) \equiv \int_{0}^{t} d \tau X_{\mathrm{RTN}}(\tau)
$$

one can show that the dephasing factor takes the form [23]

$$
\begin{aligned}
D\left[\left\{X_{\mathrm{RTN}}(t)\right\}\right] & \equiv\left|\mathbb{E}\left[\exp \left(-2 i \omega_{0} \mathcal{X}_{\mathrm{RTN}}(t)\right)\right]\right| \\
& =e^{-\gamma t}\left[\cosh (v t)+\frac{\gamma}{v} \sinh (v t)\right],
\end{aligned}
$$

with $v=\left(\gamma^{2}-4 \omega_{0}^{2}\right)^{1 / 2}$. These explicit expressions allow us to estimate the dephasing factor of Eq. (4) and study its monotonicity properties as a function of time. As we discuss in Sec. V these estimates are indeed confirmed by the experimental results and validate the theoretical analysis. It appears, in particular, that while the dephasing due to OrnsteinUhlenbeck noise $D\left[\left\{X_{\mathrm{OU}}(t)\right\}\right]$ is a decreasing function of time for any value of $\gamma$ and $\sigma$, the contribution corresponding to the random telegraph noise $D\left[\left\{X_{\mathrm{RTN}}(t)\right\}\right]$ can also exhibit an oscillating behavior [24]. Note that both processes are examples of Markovian colored noise and have an exponentially decaying correlation function; namely [20],

$$
\mathbb{E}\left[X_{\mathrm{OU}}(t) X_{\mathrm{OU}}(s)\right]=\frac{\sigma^{2}}{2 \gamma} \exp (-\gamma|t-s|),
$$

and

$$
\mathbb{E}\left[X_{\mathrm{RTN}}(t) X_{\mathrm{RTN}}(s)\right]=\exp (-2 \gamma|t-s|) .
$$

To consider a classical non-Markovian process, still retaining the property of Gaussianity, we refer to Eq. (5). The relevant quantity is again $D[\{Y(t)\}]$, which can be evaluated by exploiting the fact that $Y(t)$ is still Gaussian and relying on the properties of the Ornstein-Uhlenbeck process. The result reads

$$
\begin{aligned}
D[\{Y(t)\}] & =\left|\mathbb{E}\left[\exp \left(-2 i \omega_{0} \mathcal{Y}(t)\right)\right]\right| \\
& =\exp \left\{-\omega_{0}^{2} \sigma^{2} \frac{(\gamma-\kappa)^{2}-\left(\gamma e^{-\kappa t}-\kappa e^{-\gamma t}\right)^{2}+\gamma \kappa\left(2 e^{-(\gamma+\kappa) t}-e^{-2 \gamma t}-e^{-2 \kappa t}\right)}{\gamma \kappa(\gamma-\kappa)^{2}(\gamma+\kappa)}\right\},
\end{aligned}
$$

where according to Eqs. (7) and (9) we have denoted the integrated process as $\mathcal{Y}(t) \equiv \int_{0}^{t} d \tau Y(\tau)$. The dephasing factor shows a monotonic decaying behavior for all possible values of the constants $\gamma$ and $\kappa$, friction coefficient of the OrnsteinUhlenbeck and rate appearing in the stochastic differential equation (5), respectively.

The last process that we consider is the solution of Eq. (6), which is neither Gaussian nor Markovian due to the fact that the driving noise is colored and non-Gaussian. The evaluation of the corresponding dephasing factor

$$
D[\{Z(t)\}]=\left|\mathbb{E}\left[\exp \left(-2 i \omega_{0} \mathcal{Z}(t)\right)\right]\right|,
$$

with $\mathcal{Z}(t) \equiv \int_{0}^{t} d \tau Z(\tau)$, calls for a numerical evaluation since we can no longer exploit the important simplification in the evaluation of the characteristic function warranted for Gaussian processes. In particular, as confirmed by the experiment, it appears that depending on the parameter values also in this case an oscillating behavior can show up. It thus appears that, in this context, non-Markovianity of the quantum dynamics appears when the relevant classical process is non-Gaussian, rather then being related to a lack of the Markov property.

In various theoretical papers and experimental implementations [25] it has been shown that the appearance of quantum non-Markovianity in situations in which the environmental interaction can be characterized by a spectral density is typically related to a nontrivial peak structure of the relevant frequency spectrum. In this respect it is natural to investigate also in the present framework the relationship between spectral properties of the noise and features of the quantum dynamics. For both Ornstein-Uhlenbeck and random telegraph noise the spectrum has a Lorentzian shape centered on zero, corresponding to the exponential decay of the two-time correlation function. The correlation function of the process $Y(t)$ takes instead the form

$$
\begin{aligned}
\mathbb{E}[Y(t) Y(s)] & \left(\frac{\sigma}{\gamma-v}\right)^{2}\left\{\frac{\gamma}{2}\left(e^{-\gamma|t-s|}-e^{-\gamma(t+s)}\right)\right. \\
& +\frac{v}{2}\left(e^{-v|t-s|}-e^{-\nu(t+s)}\right) \\
& \left.+\frac{\gamma v}{\gamma+v}\left(e^{-v t-\gamma s}+e^{-\gamma t-\nu s}-e^{-v|t-s|}-e^{-\gamma|t-s|}\right)\right\} .
\end{aligned}
$$

The process is only asymptotic stationary, with associated power spectrum

$$
S(\omega)=\frac{\sigma^{2}}{2 \pi} \frac{\omega^{2}}{\left(\gamma^{2}+\omega^{2}\right)\left(\kappa^{2}+\omega^{2}\right)},
$$

featuring a double-peaked structure and a dip at small frequencies. The same feature is shared by the spectrum of the process $Z(t)$ arising as a solution of Eq. (6), which can be evaluated numerically and is shown in Fig. 1.

Despite the nontrivial structure of the power spectrum, as follows from Eq. (11) the trace distance still exhibits 


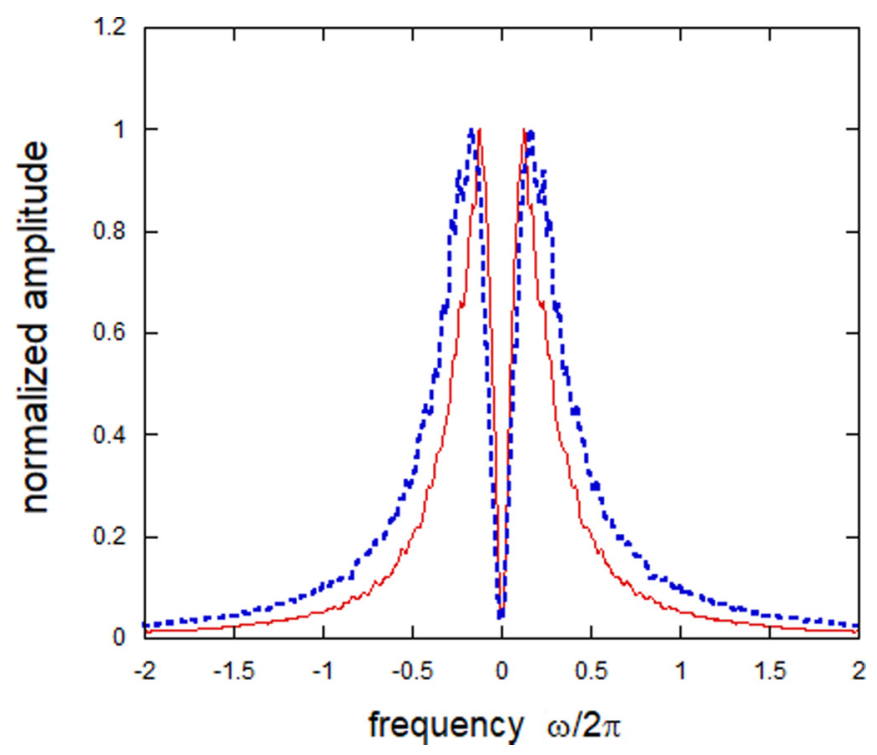

FIG. 1. Spectrum of the process $Z(t)$ obtained by the numerical evaluation of the stochastic differential equation (6) for the case $\mu=$ 0.5 (solid red line) and $\mu=1$ (dashed blue line). The coefficient $\gamma$ characterizing the RTN is set to 0.5 .

a monotonically decaying behavior, reflecting a Markovian dynamics, for the $Y(t)$ process, while oscillations may be present for the $Z(t)$ process. It therefore appears that in this context the correlation function of the classical process and the associated power spectrum does not embody the relevant

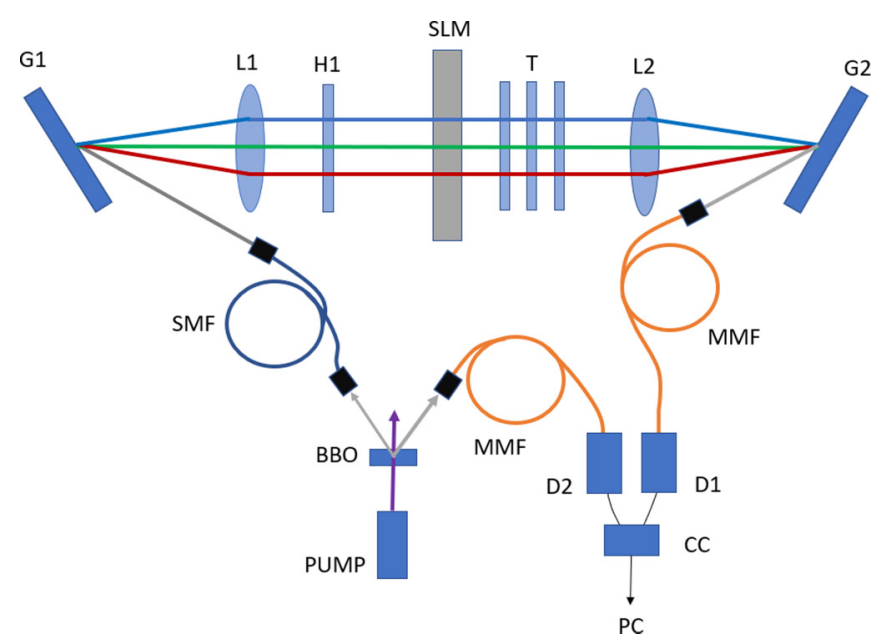

FIG. 2. Schematic diagram of our apparatus. A couple of frequency-entangled photons is generated via parametric downconversion (PDC) through a BBO crystal, using a $405.5 \mathrm{~nm}$ laser diode as pump. One photon is sent via a multimode fiber (MMF) to the single-photon detector D2. The other is sent through a single-spatial-mode and polarization-preserving fiber (SMF) to the $4 \mathrm{~F}$ system (composed by two diffraction gratings G1-G2 and two lenses L1-L2). The initial state of the photon is prepared by the half-wave plate $\mathrm{H} 1$. $\mathrm{T}$ is the tomographic apparatus, made of a quarter-wave plate, a half-wave plate, and a polarizer. The photon is then sent through a MMF to the single-photon detector D1. Finally, an electronic device measures the coincidence counts (CC) and sends them to the computer (PC).

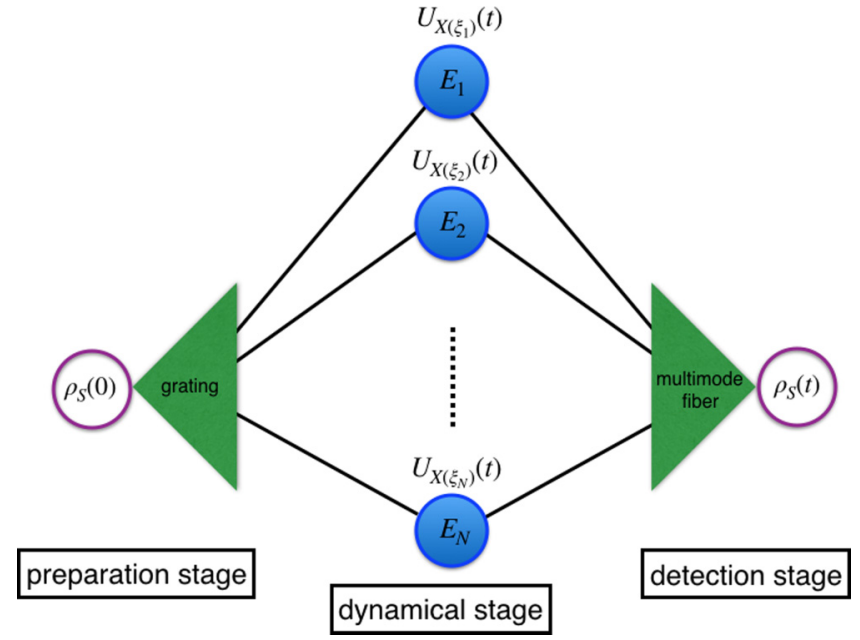

FIG. 3. Logical scheme of the experimental setting. The preparation stage involves generation of the photons and spatial separation of the different spectral components via a grating. The dynamical stage involves interaction with different regions of the SLM, imprinting different phases depending on the realization of the noise associated with the region, corresponding to a Hamiltonian interaction $U_{X(\xi)}(t)$ with a fixed noise realization. The detection stage involves recombination of the different spectral components by means of a MMF and a final photon detection.

information in characterizing the memory properties of the quantum dynamics.

\section{EXPERIMENTAL IMPLEMENTATION}

The effect of a classical noise on a quantum dephasing dynamics can be experimentally investigated in a quantum optics setup. To this aim we encode the quantum degrees of freedom in the polarization state of photons and let the noise affect the phase information. Efficiently generating and averaging over the different realizations of the noise provides the major obstacle in order to experimentally study the effect of classical disturbance on a quantum dynamics. This difficulty can be overcome by exploiting a recently realized all-optical quantum simulator [26]. This apparatus allows us to obtain many realizations of the considered stochastic process in parallel and directly averages over them at the detection stage. While details of the experimental setup have been given in Refs. [4,26], we will here provide the logical scheme of the apparatus, represented in Fig. 2. The core of the apparatus is a spatial light modulator (SLM) placed in the Fourier plane between the two lenses L1 and L2 of the $4 \mathrm{~F}$ system. The SLM is a one-dimensional (1D) liquid crystal mask (640 pixels, $100 \mu \mathrm{m} /$ pixel) used to introduce a different phase (externally controlled by the computer) to each pixel, implementing the simulation of the dynamical map. This device thus imprints different phases depending on the position and on the polarization state of the incoming photon. In the experimental device photons are generated by parametric down-conversion and a suitable grating provides a spatial separation of the different frequency components. The SLM acts differently on the different spectral components, thanks to their spatial separation, and thus allows us to encode 

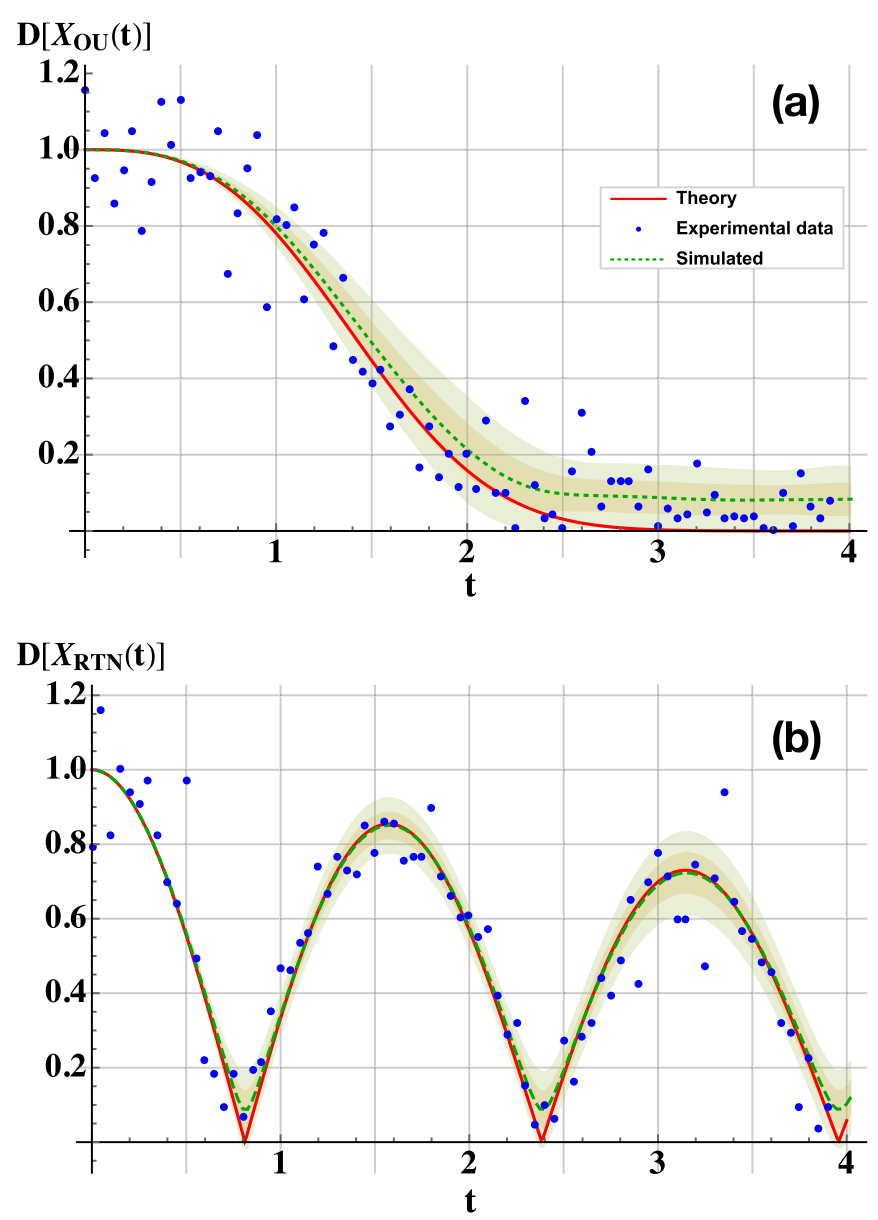

FIG. 4. Behavior of the quantum non-Markovianity quantifier $D$ defined in Eq. (4) for the case of classical Markovian processes. In panel (a) we consider the Gaussian Ornstein-Uhlenbeck process $X_{\mathrm{OU}}$ with $\gamma=0.1$ and $\sigma=0.63$. In panel (b) we consider the non-Gaussian but still Markovian random telegraph noise process $X_{\text {RTN }}$ with $\gamma=0.1$. Blue dots represent the experimental data and the red line is the analytic solution. The green dashed line is the average of 100 simulated curves, each obtained with 100 realizations of the noise. The dashed areas correspond to the $1 \sigma$ (darker) and $2 \sigma$ (lighter) intervals around the averaged coherence and $\sigma$ is the standard deviation of the sampled curves. In the first case (a) the quantum dynamics exhibits a Markovian behavior, corresponding to a monotonic decrease of coherence, while for the RTN (b) the resulting quantum dynamics is non-Markovian. Time is measured in inverse units of the rate appearing in the stochastic differential equation obeyed by the process considered.

in parallel different realizations of the noise. This experimental setup further allows us to perform the average over the realizations of the noise by collecting the different spatial components through the lens L2 and the grating G2 into a multimode fiber (MMF). The detection stage is in fact performed after recollecting the signal via the MMF, so that one averages over the spectral components and therefore the different realizations of the noise. We observe that the parametric down-conversion (PDC) spectrum is selected by the limited width of the $H 1$ plate mount. For this reason we are limited to use $n=100$ out of the 640 pixel available on the SLM
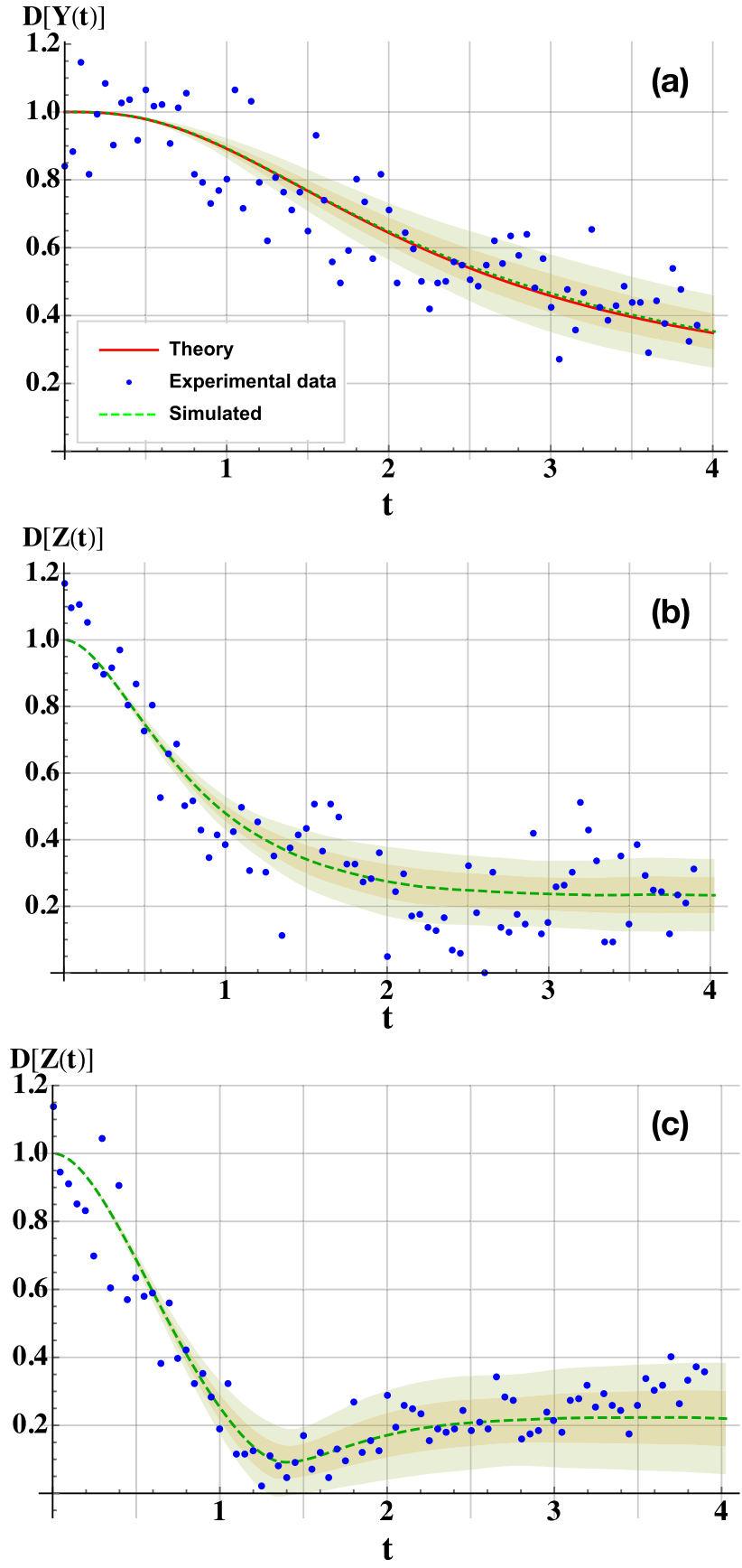

FIG. 5. Same non-Markovianity quantifier $D$ shown in Fig. 4 for the case of classical non-Markovian processes. In panel (a) we consider the Gaussian but non-Markovian processes $Y(t)$ with $k=1$. In panel (b) we consider the non-Gaussian and nonMarkovian process $Z(t)$ with $\mu=1$. In panel (c) we consider the same process $Z(t)$ with $\mu=0.5$. In the first two panels the quantum dynamics exhibits a Markovian behavior, corresponding to a monotonic decrease of coherence, at variance with the classical property. In the last panel, instead, one also has quantum revivals corresponding to a non-Markovian behavior. As in Fig. 4, the blue dots represent the experimental data and the red line represents the analytic solution, when it exists. The green dashed line is the averaged non-Markovianity and the shaded areas correspond to $1 \sigma$ and $2 \sigma$ regions around the mean value. Time is measured in inverse units of the rate appearing in the stochastic differential equation obeyed by the considered process. 
(which corresponds to 100 realizations in parallel with the noise).

As shown in the logical scheme of Fig. 3, this simple experimental setting nicely reproduces the framework considered in Ref. [15]; namely, the description of the overall reduced dynamics arising as a mixture of Markovian dynamics. In the present case in particular, the system dynamics which get mixed are given by unitary maps $U_{X(\xi)}(t)$, each characterized by a single realization of the stochastic process. In the experimental realization of the scheme it clearly appears how non-Markovianity arises because of the presence of degrees of freedom dynamically coupled to the observed ones and later averaged over.

\section{EFFECT OF NOISE ON THE QUANTUM DYNAMICS}

We here report about the experimental results for the realizations of the different kind of noise considered in Sec. III, using the apparatus described in Sec. IV. To generate the different noises we numerically solved the stochastic differential equations considered in Sec. III. The obtained values have been passed over to the SLM so as to affect the phase of the photons according to the dynamics given by Eq. (1).

The values obtained in correspondence with the different realizations have been encoded in different regions of the SLM, thus allowing for an easy implementation of the average as depicted in the logical scheme of Fig. 3. Given that the aim of the work is the comparison between non-Markovianity of the quantum dynamics and the features of the classical noise, for each kind of noise we have studied the behavior of the trace distance as a function of time.

We keep track of time by encoding in the SLM the different values of the processes at discretized times with step of order 1 in inverse units of the rate appearing in the stochastic differential equation characterizing the given process. As discussed in Sec. II B, we consider the quantity $D$ defined in Eq. (4) as quantifier of the non-Markovian features of the dynamics, which in particular fixes the behavior of the coherences. In Fig. 4 we show the experimental data referring to the quantum signature of non-Markovianity for two classical Markovian processes; namely, Ornstein-Uhlenbeck $D\left[\left\{X_{\mathrm{OU}}(t)\right\}\right]$ and random telegraph noise $D\left[\left\{X_{\mathrm{RTN}}(t)\right\}\right]$. While the former quantity is monotonically decreasing, the latter clearly shows a damped oscillating behavior, corresponding to a quantum non-Markovian behavior. Note that both processes have a power spectrum of the form (13). While both processes are classically Markovian, only Ornstein-Uhlenbeck is Gaussian. The theoretical and numerical previsions are in very good agreement with the experimental data (see also the shaded regions in Fig. 5).

We further consider two nonstandard classical processes obtained as a solution of the stochastic differential equations (5) and (6). The process $Y(t)$ is Gaussian but classically non-Markovian. Despite these properties and the nontrivial power spectrum given by Eq. (13), as shown in Fig. 5 the quantity $D[\{Y(t)\}]$ is monotonically decreasing in time. Again the experimental points are in agreement with the analytical estimate (11). In the case of $D[\{Z(t)\}]$, there exist values for the parameters that make revivals of the trace distance appear. We highlight again that the structured spectrum of both the $Y(t)$ and $Z(t)$ processes cannot be directly connected to memory effects. For such non-Gaussian processes the experimental points are compared with the results obtained via a numerical simulation of the process, further allowing us to obtain its power spectrum shown in Fig. 1. Again the classical non-Markovianity of the process is not reflected in the quantum signature.

\section{CONCLUSIONS AND OUTLOOK}

We address the quantum non-Markovianity of a singlequbit dephasing map in terms of the Markovianity of the stochastic process generating the noise. In particular, we considered four random processes with different Gaussianity and Markovianity traits. We showed that the Markovianity of the classical stochastic process does not affect the information backflow to the system, i.e., classical lack of Markovianity is not directly related to memory effects. However, we showed evidence that the non-Gaussianity of the noise can be related with oscillations of the trace distance.

\section{ACKNOWLEDGMENTS}

The authors acknowledge support from the Joint Project "Quantum Information Processing in Non-Markovian Quantum Complex Systems" funded by FRIAS, University of Freiburg, and IAR, Nagoya University, from the FFABR project of MIUR and from the Unimi Transition Grant H2020. B.V. gratefully acknowledges useful discussions with Alberto Barchielli and Matteo Gregoratti.
[1] Thermodynamics in the Quantum Regime, edited by F. Binder, L. A. Correa, C. Gogolin, J. Anders, and G. Adesso, Fundamental Theories of Physics, Vol. 195 (Springer, Berlin, 2018).

[2] A. W. Chin, S. F. Huelga, and M. B. Plenio, Phys. Rev. Lett. 109, 233601 (2012).

[3] J. F. Haase, A. Smirne, S. F. Huelga, J. Kołodynski, and R. Demkowicz-Dobrzanski, Quantum Meas. Quantum Metrol. 5, 13 (2018).

[4] A. Smirne, S. Cialdi, G. Anelli, M. G. A. Paris, and B. Vacchini, Phys. Rev. A 88, 012108 (2013).
[5] A. Rivas, S. F. Huelga, and M. B. Plenio, Rep. Prog. Phys. 77, 094001 (2014).

[6] H.-P. Breuer, E.-M. Laine, J. Piilo, and B. Vacchini, Rev. Mod. Phys. 88, 021002 (2016).

[7] I. de Vega and D. Alonso, Rev. Mod. Phys. 89, 015001 (2017).

[8] B. Vacchini, A. Smirne, E.-M. Laine, J. Piilo, and H.-P. Breuer, New J. Phys. 13, 093004 (2011).

[9] B. Vacchini, J. Phys. B: At., Mol. Opt. Phys. 45, 154007 (2012). 
[10] G. Guarnieri, A. Smirne, and B. Vacchini, Phys. Rev. A 90, 022110 (2014).

[11] M. Gessner, M. Ramm, H. Haeffner, A. Buchleitner, and H.-P. Breuer, Europhys. Lett. 107, 40005 (2014).

[12] L. Li, M. J. Hall, and H. M. Wiseman, Phys. Rep. 759, 1 (2018).

[13] A. Smirne, D. Egloff, M. G. Díaz, M. B. Plenio, and S. F. Huelga, Quantum Sci. Technol. 4, 01LT01 (2019).

[14] G. Lindblad, Commun. Math. Phys. 65, 281 (1979).

[15] H.-P. Breuer, G. Amato, and B. Vacchini, New J. Phys. 20, 043007 (2018).

[16] H.-P. Breuer, E.-M. Laine, and J. Piilo, Phys. Rev. Lett. 103, 210401 (2009).

[17] E.-M. Laine, J. Piilo, and H.-P. Breuer, Phys. Rev. A 81, 062115 (2010).

[18] D. Chruściński and F. A. Wudarski, Phys. Lett. A 377, 1425 (2013).
[19] N. Megier, D. Chruściński, J. Piilo, and W. T. Strunz, Sci. Rep. 7, 6379 (2017).

[20] C. W. Gardiner, Handbook of Stochastic Methods for Physics, Chemistry and the Natural Sciences, Springer Series in Synergetics, Vol. 13 (Springer-Verlag, Berlin, 2004), 3rd ed.

[21] R. F. Fox, J. Math. Phys. 18, 2331 (1977).

[22] A. Hernández-Machado and M. San Miguel, J. Math. Phys. 25, 1066 (1984).

[23] B. Abel and F. Marquardt, Phys. Rev. B 78, 201302 (2008).

[24] C. Benedetti, M. G. A. Paris, and S. Maniscalco, Phys. Rev. A 89, 012114 (2014).

[25] B.-H. Liu, L. Li, Y.-F. Huang, C.-F. Li, G.-C. Guo, E.-M. Laine, H.-P. Breuer, and J. Piilo, Nat. Phys. 7, 931 (2011).

[26] S. Cialdi, M. A. C. Rossi, C. Benedetti, B. Vacchini, D. Tamascelli, S. Olivares, and M. G. A. Paris, Appl. Phys. Lett. 110, 081107 (2017). 